\title{
LABEL MAPS FUSION FOR THE MARGINAL SEGMENTATION OF MULTI-COMPONENT IMAGES
}

\author{
S. Alkama*, Y. Chahir $\dagger$ D. Berkani $i^{\ddagger}$
}

\begin{abstract}
In this paper, we propose a new technique for merging the label maps obtained by the marginal segmentation of a multi-component image. In the marginal segmentation, each component of the multi-component image is independently segmented by labeling the pixels of the same class with the same label. Therefore the number of label maps corresponds to the number of components in the image. It is then necessary to merge them in order to have a single label map, i.e. a single segmented image. In the most merging techniques, the compatibility links between these maps are performed a priori by making the correspondences between their labels. However the various components are segmented and labeled independently, label maps are considered as independent sources. It is then difficult to establish the relationship compatibilities between labels. The method we propose does not a priori assume any compatibility links. The label maps are combined by superposition. Unfortunately, an over-segmentation is produced. To cope with this problem, the insignificant regions and classes are eliminated. Finally, classes are grouped by using hierarchical agglomerative clustering algorithm. Tests performed on color and satellite images show the effectiveness of this method and its superiority compared to the vector segmentation. The self-organizing map is used during the segmentation process in both marginal and vector segmentations.
\end{abstract}

Key words: marginal segmentation, fusion of label maps, multi-component image, Self Organization Maps

Received: July 13, 2014

DOI: $10.14311 /$ NNW.2015.25.021

Revised and accepted: March 8, 2015

\section{Introduction}

Image segmentation is a field of research which is still expanding. The techniques of image segmentation were first developed for gray-level images (monochrome or

\footnotetext{
* Sadia Alkama - Corresponding author, Department of Automatics, Mouloud Mammeri University, 15000 Tizi-Ouzou, Algeria, E-mail: sadia_alkama@mail.ummto.dz

${ }^{\dagger}$ Youssef Chahir, GREYC-UMR CNRS 607, Campus II, BP 5186, Caen University, 14032 Caen Cedex, France, E-mail: youssef .chahir@unicaen.fr

$\ddagger$ Daoud Berkani, Department of Electronics, Polytechnic National School, 16200 El Harrach, Algeria, E-mail: daoud.berkani@enp.edu.dz
} 
mono-component), then they were extended to the multi-component images which have a higher dimension and are provided by more complex vision systems. The multi-components images provided by these sensors are used in various sectors such as in medical field, remote sensing, quality control, etc. These images can be color, multi-spectral, medical, etc.

The segmentation is a critical step in image processing because it affects the quality of the interpretation and of the decision-making. It consists in regrouping the pixels of the image in regions according to their characteristics or features. Several approaches can be used for this purpose. The techniques based on the pixel classification approach consist in assigning a class number to each pixel of the image. The result of this operation is a segmented image called a label map where each pixel is represented by one label which corresponds to the class number in which this pixel has been assigned. Note that the problems of segmentation and classification are closely linked and both words can be used to mean the same thing as all pixels within the same class and which are connected form a region of the image.

Our interest in this paper is the multi-component image segmentation. Three approaches are used to segment the multi-component images $[12,33,41]$. The first one, called scalar approach, consists in segmenting a single monochrome image obtained by merging all components of the multi-component image. The so-called vector approach considers each pixel as a vector where each element corresponds to a component value and only one processing is applied to this image. The approach described as marginal consists in applying the same segmentation technique independently to each component of the image. This last technique provides a set of label maps where the number of maps is equal to the number of components in the image. These label maps must be merged in order to provide a single label map.

Contrary to the vector approach where many works deal with this field $[1,6,19$, $21,26,30,49,50]$, few works has been devoted to the marginal segmentation where the major difficulty lies in the fusion of label maps. The works dealing with label maps fusion often merge the binary maps which are edge/no-edge maps obtained by using a contour detection. The merge of edge/no-edge maps is often performed by logical operators such as "or" and "and" $[8,18,43]$ or by statistical analysis of correspondences between these maps [16].

When a label map contains more than two labels the merging problem becomes more difficult. Some authors $[10,32]$ perform this fusion by using the evidence theory which has been originally introduced by Dempster [14], and further developed by Shafer [45]. One difficulty of this theory is in the choice of the modeling mass functions [9]. Another problem with this technique is in establishing the compatibility links between the labels of various maps. In fact, the various components of the image are segmented and labeled independently since the label maps are considered as independent sources. There is no guarantee that the same label is assigned to the same area of interest in these different maps. To complete the fusion by the evidence theory, the correspondence between the labels must be made in advance.

The fusion of the label maps can be performed also by using the majority-vote rule [39]. This technique starts by establishing the correspondence of labels in the different label maps. Then, the fusion of these label maps, which are semantically 
Alkama S., Chahir Y., Berkani D.: Label maps fusion for the marginal...

equivalent, is performed by assigning to each pixel in the final map the label that occurs most frequently in the original label maps.

Other authors have treated the fusion of label maps differently $[20,22,31,33$, 46, 47]. For example, Kurugollu et al. [31] propose a semi-marginal color image segmentation by a multi-thresholding of the RG, RB and BG histograms. The fusion of three label maps is conducted in two successive steps. The first step performs the concordance of labels in the three maps by assigning the same value for the labels having a maximum of occurrences. The next step performs the maps fusion by assigning for each pixel label that is most repeated in its $5 \times 5$ neighborhood. Lezoray and Charrier [33] use a morphological clustering, on the two-dimensional histograms RG, RB and BG, to create three label maps. Then, they eliminate in each map the regions which are sized less than a structuring element of size $3 \times 3$. Thereafter, they superpose these maps. The fusion map thus generated produces a large number of the labels and regions. A region merging technique is then performed by using a spatial adjacency graph where the adjacent and similar regions are merged.

The fusion of label maps is also involved in the combination of several different segmentations of the same image produced by different parameter settings of the same segmentation algorithm or by different segmentation algorithms $[15,20,24$, 44]. Combining multiple segmentations can be viewed as a special case of the cluster ensemble problem, i.e., the concept of combining multiple data clustering for the improvement of the final clustering result [38]. For example, Franek et al. [15] produce $N$ segmentations of an image by varying some parameters or by using different segmentation algorithms. They after generate a super-pixels image (regions image) by combing these $N$ segmentation label maps. They eliminate small regions before they use an ensemble clustering method to reduce the number of objects. Because of eliminating small super pixels before some pixels are unlabeled. These pixels are simply merged to the neighboring region with the smallest color difference.

The fusion of label maps is also used in the combination of the segmentation maps obtained by different segmentations on a same image with different color spaces $[11,17,37]$. The fusion model proposed in [37] and [17] combines the individual input segmentations by applying a $K$-means algorithm where each pixel is characterized by a set of local label histogram values given by each input segmentation. The same strategy with the spatial FCM clustering, instead of $K$-means, is used in [11].

We propose in this paper a new fusion technique of the label maps. This procedure is simple to implement, fast and can be used in various computer vision applications. It is fully automatic contrary to other techniques that perform the fusion by using the evidence theory or the ensemble clustering approach. Recall that to complete the fusion by using the evidence theory or the majority-vote, the correspondence between the labels must be made in advance, usually by an expert user and rarely is it performed automatically. In the proposed fusion procedure, this tedious step is eliminated and the fusion is fully automatic and no correspondence between the labels is needed. Furthermore, some techniques like in $[15,33]$ remove small regions in each label maps before the fusion step. This procedure is then computed as many times as there are components in the image. After 
combining the maps, a new removing procedure of the small regions can also be used. This downstream processing is computationally expensive and removes regions which will be removed in the upstream processing. To save time and because an upstream processing is more relevant than a downstream processing, in our proposed method only an upstream processing is performed. Indeed, the downstream processing processes each component independently of the other components, while the upstream processing involves the interaction of spectral components because it did on the combined component. The other originality of the proposed fusion procedure lies in how the pixels belonging to the small (insignificant) regions and small (insignificant) classes removed, are assigned to the remaining significant classes.

Before the fusion, the classification algorithm SOM (Self Organization Maps) is used to segment independently each component of the multi-component image. This algorithm has been widely used and demonstrated its effectiveness in the field of the image segmentation $[2,5,25,27,35,42]$. In the proposed fusion procedure, the label maps are combined into a single map by assigning for the same "label combination" of label maps the same label in this resulting map. Conversely, different "label combinations" will have different labels in the resulting map. In this new and single map, the number of regions and classes (labels) are very high. Insignificant regions and classes are then eliminated by assigning efficiently their corresponding pixels to others classes which are considered as significants. Finally, the hierarchical agglomerative clustering algorithm is used to group the remaining classes until reach the desired number of classes. The results obtained by this marginal segmentation technique are compared with those obtained by a vector segmentation technique using the same SOM algorithm.

The remainder of this paper is organized into five sections. The proposed marginal segmentation is cursory described in the Section 2. Section 3 is devoted to the SOM classification algorithm used in the first phase of proposed segmentation. The steps of the proposed fusion method are detailed in Section 4. The different results, their assessments and a discussion are given in Section 5. We conclude with a conclusion in Section 6 .

\section{Proposed marginal segmentation}

In the marginal segmentation approach, the components of the image are processed separately. The number of components depends on the kind of the multi-component image. For example, it is equal to three in the case of the color images and it can be higher in the case of the multi-spectral images.

The marginal segmentation is made in two successive phases which are the monochrome independent segmentation of each component and the fusion of label maps obtained in the previous phase.

In the first phase, any gray-level image segmentation technique can be used. In this paper, the SOM algorithm is chosen to segment each component of the image because of its effectiveness proved in the field of the image segmentation $[5,25,27,42]$. This algorithm is presented in the next section. Each component of the multi-component image is segmented by the SOM algorithm independently of others components. A label map of each component is then generated by assigning to the pixels of the same class a same label and to the pixels belonging to different 
Alkama S., Chahir Y., Berkani D.: Label maps fusion for the marginal...

classes different labels. We obtain then as much label maps as there has components in the image. For example, for the color images, we obtain three label maps.

The second phase of this strategy is very delicate. Some works deal with the label maps fusion $[8,10,31,33]$. The most of them need a priori the knowledge of compatibility links between the label maps. In this paper we propose a new fusion technique to merge the label maps. This technique do not a priori assume any compatibility link between labels. It consists of four steps which are illustrated in Fig. 1. To distinguish the different changes in these steps, an illustration on a color image is presented. The detail of this phase is given in Section 3. Note that the label maps are displayed as color images where each label (i.e. class) is represented by the average color of all pixels having this label (i.e. assigned to this class) and the boundaries are deliberately indicated in bold by white color.

\section{Segmentation by self-organizing maps}

Topographic maps or self-organizing maps (SOM) are neural networks that are used for unsupervised learning. They were introduced for the first time by T. Kohonen in $1981[28,29]$. Since then, many works deal with the SOM algorithms especially in the image processing and image segmentation $[2,3,35,48]$. The most important feature of these maps is their ability to self-organize observations into groups.

Before developing the principle of self-organizing maps, it is necessary to introduce some notations. Let $\Upsilon$ be the set of the observations of $D$ dimensions, $\Upsilon \subset \mathbb{R}^{D}$. The self-organizing map is a neural network that consists of two layers (see Fig. 2):

- An input layer, which is the subset $Y=\left\{y_{i} ; i=1, \ldots, n\right\}$ of $\Upsilon$ consisting of $n$ elements of a learning set. In our case, $Y$ represents a multi-component image containing the pixels $y_{i}$ characterized by $D$ features. It can also represent one component of a multi-component image in the case of a marginal segmentation, in this case $D$ is equal to 1 . In this input layer, all states of all neurons are then forced to the values of the observations $y_{i}$.

- A $2 \mathrm{D}$ computational layer, noted $\varphi$, which is formed of lattice neurons that are forming the map. This neural set is denoted $W=\left\{\omega_{c} ; c=1, \ldots, p\right\}$ and formed by $p$ vectors of $\Upsilon$, which are $D$-dimensional. Each of these neurons is connected to all elements of the input layer.

Let $F$ be an allocation function defined as an application of $Y$ to the set $\{1, \ldots, p\}(F: Y \rightarrow\{1, \ldots, p\})$. This function $F$ is used to assign to each input vector $y_{i} \in Y$ a neuron with the index $c$ selected from the set $\{1, \ldots, p\}$ and designated by the weight vector $\omega_{c}$, such as the vector $\omega_{c}$ is the most similar to $y_{i}$.

$$
F\left(y_{i}\right)=\arg \min _{c}\left\|y_{i}-\omega_{c}\right\|^{2} .
$$

During the learning phase of the network, the weight $\omega_{c}$ of the winning neuron $c$ is modified as well as weights of the neurons of its neighborhood. The distances $\delta(c, r)$ which link the neuron $c$ to the other neurons $r$ of the map allow to vary the relative influence of the various neurons [29]. 


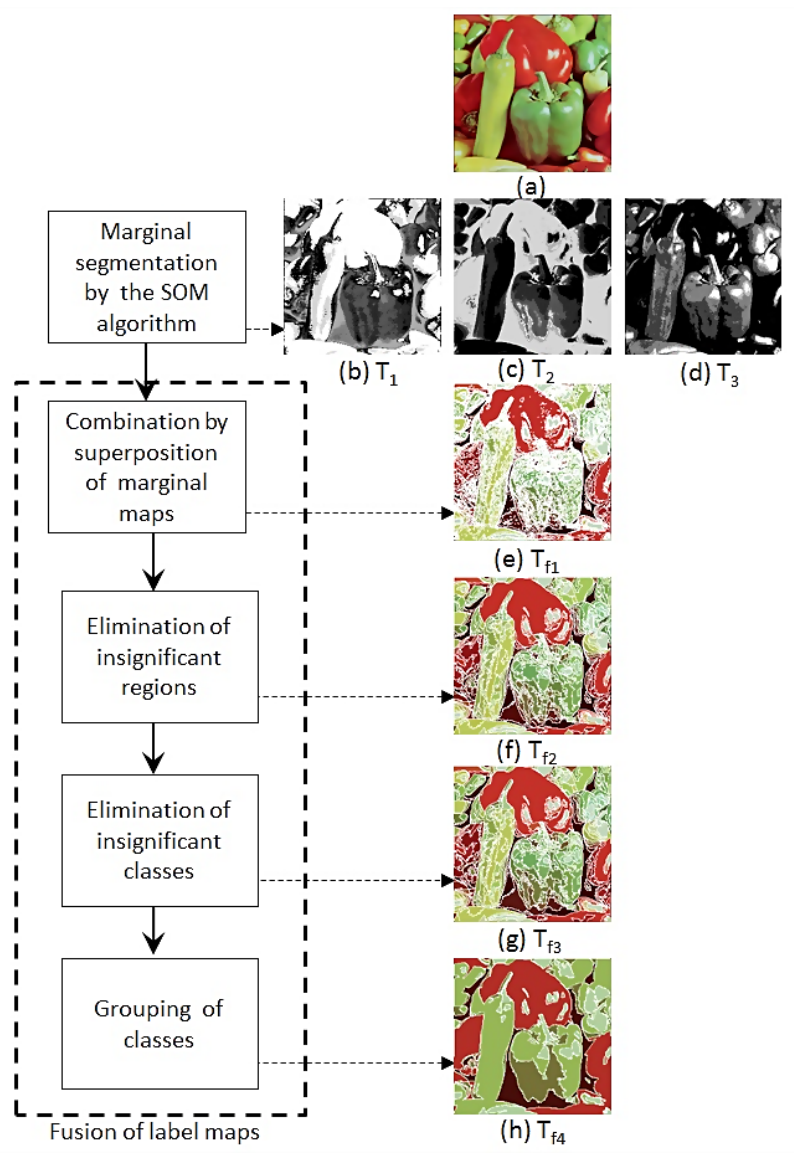

Fig. 1 General scheme of the proposed marginal segmentation approach: (a) Original image. Marginal segmentation into 7 classes of the components: (b) red (c) green (d) blue. (e) Combination map (187 classes, 9063 regions). (f) Label map without insignificant regions (90 classes, 388 regions). (g) Label map without insignificant classes (73 classes, 314 regions). (h) Final label map (7 classes, 86 regions).

Recall that the distance $\delta$ in $\varphi$ of any pair of neurons $(c, r)$ of this map is defined as the length of the shortest path between $c$ and $r$ on $\varphi$. For example, in Fig. 2 the distance between $\mathrm{r}$ and $\mathrm{c}$ is $\delta(c, r)=4$.

This relative influence of the various neurons is quantified by the neighborhood function $V^{T}(\delta(c, r))$ which forces the weights of neurons located in the neighborhood of neurone $c$ to get their values near to the input vector $y_{i}$. The more a neuron is far from the best matching neuron, the less the variation is important. The function of neighborhood used in this paper is parameterized by a temperature factor $T$ in order to take into account the size of the neighborhood and is given by

$$
V^{T}(\delta)=\exp \left(-\frac{\delta}{T}\right) .
$$


Alkama S., Chahir Y., Berkani D.: Label maps fusion for the marginal...

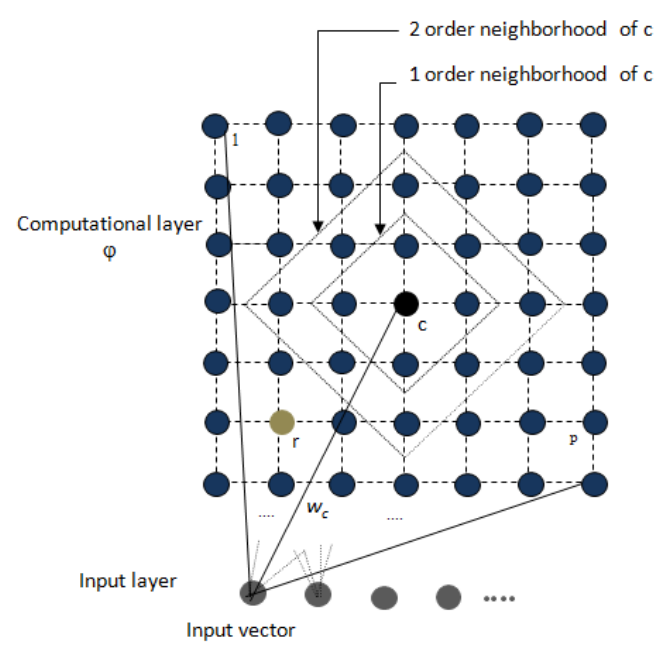

Fig. 2 Representation of a $2 D$ topographic map of Kohonen.

The self-organizing map algorithm minimizes the cost function noted $J_{\text {som }}^{T}[29]$ given by

$$
J_{\text {som }}^{T}(W)=\sum_{y_{i} \in Y} \sum_{c \in C} V^{T}\left(\delta\left(c, F\left(y_{i}\right)\right)\right)\left\|y_{i}-\omega_{c}\right\|^{2} .
$$

The minimum is obtained by setting to zero the derivative of $J_{\text {som }}^{T}(W)$. In the stochastic version of Kohonen, it is not required to find the global minimum of $J_{\text {som }}^{T}(W)$, it is enough to decrease its value. Thus, at the $t$-th iteration, the weight $c$ of a neuron is modified according to

$$
\omega_{c}^{t}=\omega_{c}^{t-1}+\mu^{t} \frac{\partial J_{\mathrm{som}}^{T}}{\partial \omega_{c}^{t-1}},
$$

where

$$
\frac{\partial J_{\mathrm{som}}^{T}}{\partial \omega_{c}}=2 \sum_{y_{i} \in Y} V^{T}\left(\delta\left(c, F\left(y_{i}\right)\right)\right)\left(y_{i}-\omega_{c}\right) .
$$

The neuron weights are updated by using the following equation:

$$
\omega_{c}^{t}=\omega_{c}^{t-1}-\mu^{t} V^{T}\left(\delta\left(c, F\left(y_{i}\right)\right)\right)\left(\omega_{c}^{t-1}-y_{i}\right),
$$

where $\mu^{t}$ is a decreasing function according to the iterations $t$. In this work we use

$$
\mu^{t}=\frac{1}{\sqrt{t}}
$$

The Kohonen algorithm is summarized in Algorithm 1.

The function $V^{T}$ of neighborhood is parameterized by a temperature factor $T$ in order to take into account the size of the neighborhood. For large values of $T$, an observation $y_{i}$ changes a large number of neuron weights. On the contrary, for 


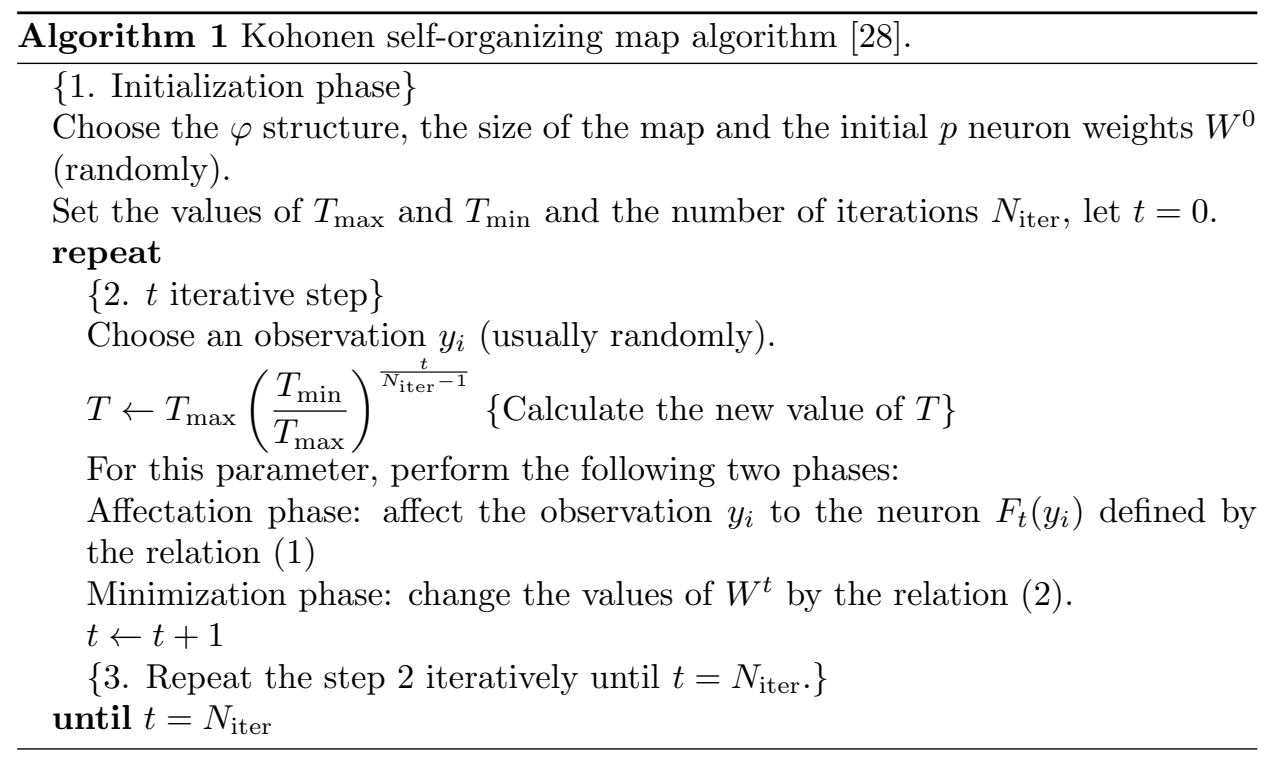

small values of $T, V^{T}(\delta(c, r))$ is negligible if $c \neq r$ : an observation influences only the calculation of the winner neuron weight, $\omega_{c}$. At the beginning of the learning process, the size of the neighborhood is fairly large, but it is made to shrink during learning. This ensures that the global order is obtained already at the beginning, whereas towards the end, as the neighborhood gets smaller, the local corrections of the neuron weights in the map will be more specific. For that, the factor $T$ decreases during learning from $T_{\max }$ to $T_{\min }$. In this paper the size of Kohonen map is $\tau \times \tau$, then $T_{\max }$ is set to $\tau$ and $T_{\min }$ to 1 (with $\tau=10$ ).

The self-organization, as we described it, does not solve classification problems. Each observation is assigned to a neuron of the map, independently of any notion of class. The classification problem is then just the labeling of each neuron of the map by a number class. The number of neurons is generally greater than the desired number of classes, the labeling can be done by grouping suitably these neurons. In this paper, we propose a grouping procedure based on the hierarchical agglomerative clustering algorithm [23]. Because of the topology of the map, only the neighbor neurons and the neighbor sub-groups of neurons in the Kohonen map are grouped.

After this grouping procedure, we obtain finally the desired number of neurons, namely $K$. Each of these neurons is represented by one number noted $k$ with $k=1, \ldots, K$. The label map is created by assigning for each pixel the number of the neuron which it is assigned (it should be noted that the word "map" in the self-organization map (SOM) should not be confused with the word "map" in the label map).

The vector segmentation by the SOM algorithm provides one label map noted $T$ while the marginal segmentation provides many label maps, each corresponds to one component of the image. In this case, they are noted $T_{i}$ with $i=1, \ldots, D$, where $D$ is the number of image components (channels). Fig. 3 shows the results of the vector segmentation and the marginal segmentation of the image "Peppers" 


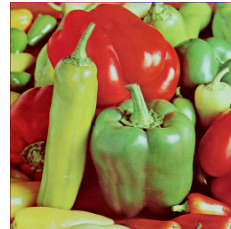

(a)

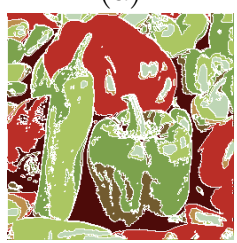

(c)

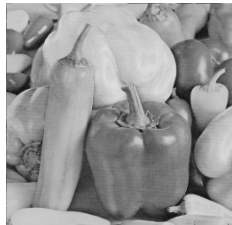

(b1)

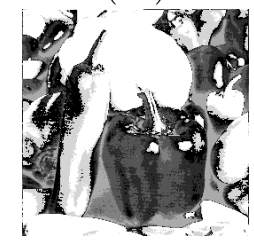

(d1)

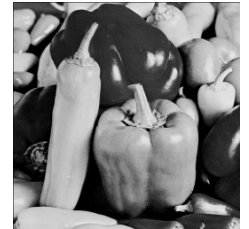

(b2)

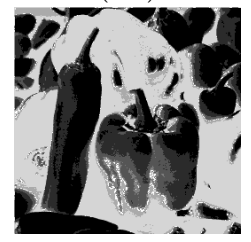

(d2)

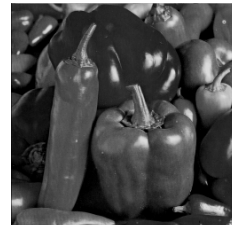

(b3)

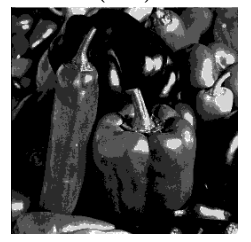

(d3)

Fig. 3 Segmentation by the SOM algorithm. (a) Original image, (b1) red component, (b2) green component, (b3) blue component, (c) T vector label map, (d1) $T_{1}$ marginal label map of the red component, (d2) $T_{2}$ marginal label map of the green component, (d3) $T_{3}$ marginal label map of the blue component.

by the SOM algorithm. The $T$ label map in this figure is displayed by affecting for each label (class) the average color of all pixels having this label (class) while the $T_{i}$ label maps are displayed in a gray levels.

\section{Fusion of label maps}

The first phase of the marginal segmentation produces $D$ label maps (see the previous Section). The second phase of the marginal segmentation consists in the fusion of these label maps. In this section we propose a new fusion technique, which consists in four steps:

1. combination by superposition of marginal maps,

2. elimination of insignificant regions,

3. elimination of insignificant classes,

4. grouping of classes.

\subsection{Combination by superposition of marginal maps}

Let us have some $D$ marginal label maps, identifying the components of some image. The goal of the first step is to create, from the label maps $T_{1}, T_{2}, \ldots, T_{D}$ obtained during the marginal segmentation phase, a new label map noted $T_{f_{1}}$, such that

$$
T_{f_{1}}=\bigvee_{i=1}^{K} T_{i},
$$

where $\bigvee$ is an operator which performs the combination by superposing the marginal label maps $T_{i}, i=1, \ldots, D$. 


\begin{tabular}{|l|l|l|l|}
\hline 1 & 1 & 2 & 2 \\
\hline 1 & 1 & 2 & 2 \\
\hline 3 & 3 & 2 & 2 \\
\hline 3 & 3 & 2 & 2 \\
\hline
\end{tabular}

(a)

\begin{tabular}{|l|l|l|l|}
\hline 2 & 2 & 2 & 3 \\
\hline 2 & 2 & 3 & 3 \\
\hline 1 & 1 & 3 & 3 \\
\hline 1 & 1 & 3 & 2 \\
\hline
\end{tabular}

(b)

\begin{tabular}{|l|l|l|l|}
\hline 3 & 3 & 3 & 1 \\
\hline 3 & 3 & 3 & 1 \\
\hline 2 & 2 & 1 & 1 \\
\hline 2 & 2 & 1 & 1 \\
\hline
\end{tabular}

(c)

$$
\begin{aligned}
& (1,2,3) \rightarrow 1 \\
& (2,2,3) \rightarrow 2 \\
& (2,3,1) \rightarrow 3 \\
& (2,3,3) \rightarrow 4 \\
& (3,1,2) \rightarrow 5 \\
& (2,2,1) \rightarrow 6
\end{aligned}
$$

\begin{tabular}{|l|l|l|l|}
\hline 1 & 1 & 2 & 3 \\
\hline 1 & 1 & 4 & 3 \\
\hline 5 & 5 & 3 & 3 \\
\hline 5 & 5 & 3 & 6 \\
\hline
\end{tabular}

(e)

Fig. 4 An example of combination of three label maps: (a) $T_{1}$ label map, (b) $T_{2}$ label map, (c) $T_{3}$ label map, (d) correspondence of the labels, (e) $T_{f_{1}}$ label map.

This combination consists in generating the same label in $T_{f_{1}}$ for the same combination of labels in the maps $T_{i}$, and different labels for different combinations. An example of the combination by superposition of three label maps $\left(T_{1}, T_{2}, T_{3}\right)$ is illustrated in Fig. 4. Each label map $T_{i}$ has three labels $(K=3)$, see Fig. 4(a), (b), (c), and the $T_{f_{1}}$ produced label map has six labels (classes), see Fig. 4(e). The correspondence between the six labels in $T_{f_{1}}$ and the combination of labels in $\left(T_{1}, T_{2}, T_{3}\right)$ is given in Fig. $4(\mathrm{~d})$.

Unfortunately, the combination by superposition as proposed here produces an over-segmentation of the image. Indeed, if we have $K$ classes in each map $T_{i}$, the combination procedure produces $M_{1}$ classes in $T_{f_{1}}$ map with $K<M_{1}<K^{D}$. This number, usually high, induces a large number of regions. For instance, in the example of Fig. 1, each $T_{i}$ label map has 7 classes while the $T_{f_{1}}$ map contains 187 classes and 9063 regions.

\subsection{Elimination of insignificant regions}

To cope with the problem of the over-segmentation, we begin by eliminating very small regions because they are assumed to be insignificant regions (holes and noises) and can't really correspond to any object in the image. Note that the regions are constituted of the adjacent pixels belonging to the same class. They are determined by using a connected component analysis procedure from the label class map $T_{f_{1}}$. The corresponding region map is noted $R$ with $R=\cup R_{i}\left(R_{i}\right.$ indicates the $i$-th region) and $R_{i} \cap R_{j}=\emptyset$ for $i \neq j$.

The insignificant regions are the areas with sizes that don't exceed $0.06 \% \times n$ pixels ( $n$ is the image size). This threshold value is chosen experimentally after several tests with different color images and satellite images. For example, for an image of $128 \times 128$ size, the threshold value is equal to 9 and for an image of $256 \times 256$ size, it is equal to 39 .

The eliminated regions are generally merged to neighbored regions. However in this paper we propose another strategy which consists in assigning the pixels of the eliminated regions to classes which contain pixels neighborhood to these eliminated regions. This new strategy is performed as follows. 
Alkama S., Chahir Y., Berkani D.: Label maps fusion for the marginal...

For each insignificant region $R_{i}$, we compute its mean color $\bar{y}_{i}$ by using the pixel values of this region,

$$
\bar{y}_{i}=\frac{1}{n_{i}} \sum_{j \in \Omega_{i}} y_{j},
$$

where $n_{i}$ is the number of pixels in the region $R_{i}$ and $\Omega_{i}$ is the set of the indices of pixels constituting the region $R_{i}$.

After that, we seek in the $T_{f_{1}}$ label map, the classes of neighborhood pixels of this region $R_{i}$. Let $\Gamma_{i}$ be the set of these classes, $\Gamma_{i}=\left\{C_{k}: C_{k}\right.$ is the class of neighboring pixels to the region $R_{i}$.

For each class $C_{k}$ of $\Gamma_{i}$ (labeled $k$ in $T_{f_{1}}$ map), we compute its mean color $\bar{m}_{k}$ as

$$
\bar{m}_{k}=\frac{1}{n_{k}} \sum_{j \in C_{k}} y_{j} .
$$

The pixels of the insignificant region $R_{i}$ are then labeled by the index of that class $C_{k}$ of $\Gamma_{i}$, which has its mean color closest to the mean color of this region. Let us denote this index as $l$. It can be obtained as

$$
l=\arg \min _{k}\left\|\bar{y}_{i}-\bar{m}_{k}\right\|^{2}
$$

At the end of this step, we get another label map noted $T_{f_{2}}$ which contains $M_{2}$ classes $\left(M_{2} \leq M_{1}\right)$ with a reduced number of regions. For the example in Fig. 1, the 187 classes in $T_{f_{1}}$ becomes 90 in $T_{f_{2}}$ and the 9063 regions in $T_{f_{1}}$ becomes 388 in $T_{f_{2}}$.

The algorithm describing this procedure is given in Algorithm 2 and an illustrative example is given in Fig. 5. Fig. 5(a) shows the label class map $T_{f_{1}}$ and Fig. 5(b) shows its corresponding region map. The region $R_{4}$ is considered as insignificant. It can be merged to one of the classes of its neighborhood among the set $\left\{C_{1}, C_{2}, C_{3}, C_{5}\right\}$. If the mean color of $C_{2}$ is supposed closest to the mean color of the region $R_{4}$, then the pixels of this region are assigned to $C_{2}$ (Fig. $5(\mathrm{c})$ ).

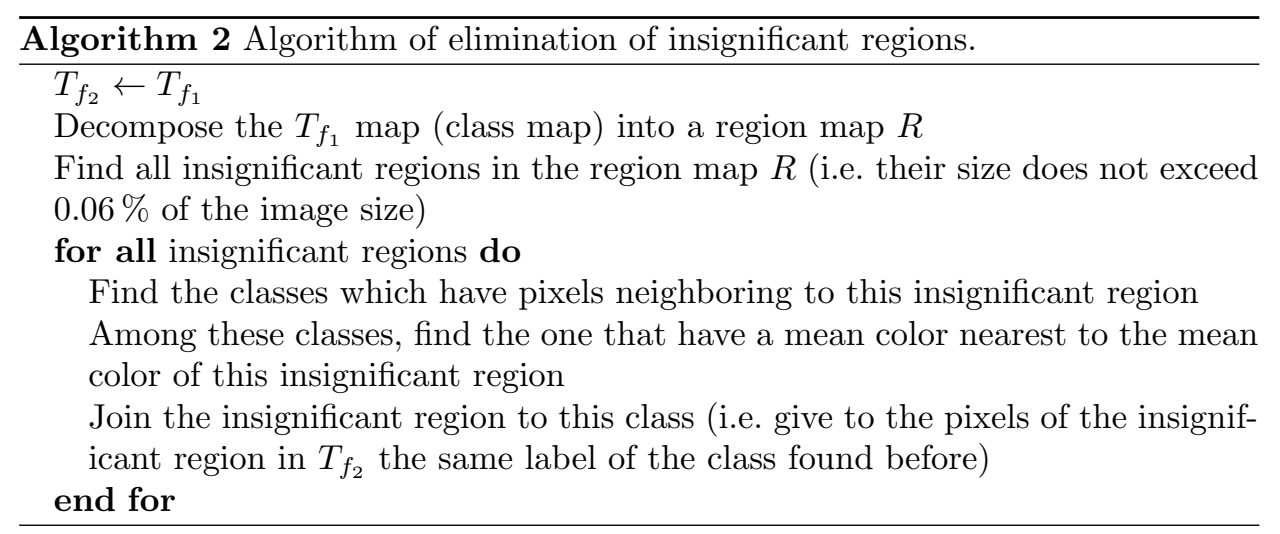




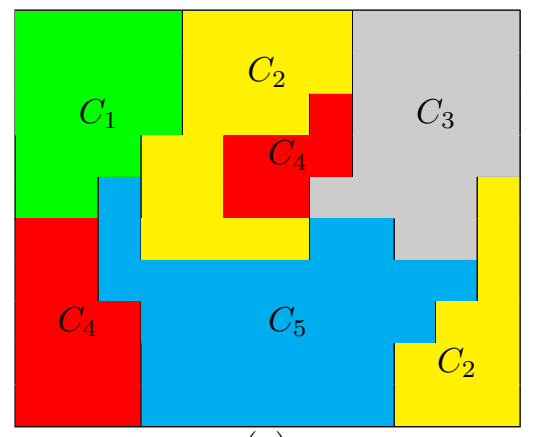

(a)

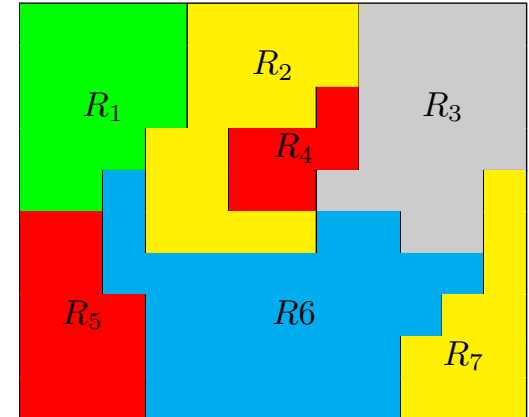

(b)

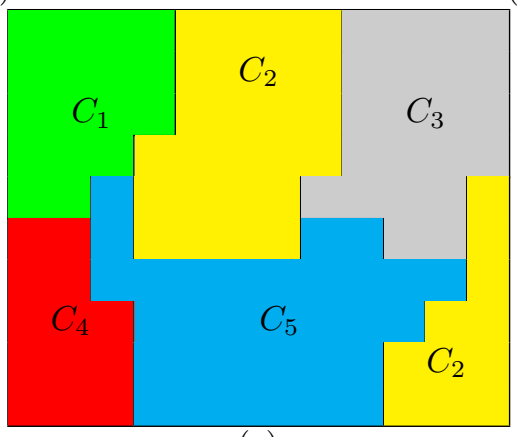

(c)

Fig. 5 Example of elimination of insignificant regions: (a) $T_{f_{1}}$ label map, (b) $R$ region map, (c) $T_{f_{2}}$ label map.

\subsection{Elimination of insignificant classes}

In the previous step, the number of regions is substantially reduced, but it can still be high. In order to reduce this number once again, we propose to eliminate all the classes which have a number of pixels relatively small. Indeed, we consider that the classes with a number of pixels less than $0.5 \% \times n$ are non-significative. This threshold value is also determined after several tests on different color and satellite images. For an image of $128 \times 128$ size, this value is equal to 81 and for an image of $256 \times 256$ size, it is equal to 327 .

The pixels of these insignificant classes are assigned to remaining classes by using the same rule described in the previous section. More precisely, the regions formed by the adjacent pixels and belonging to an insignificant class are determined. Then, the pixels of each of these regions are affected to the class of its neighborhood pixels which has a mean color closest to the mean color of this region. The results of this procedure is another label map denoted $T_{f_{3}}$, where the number of classes is $M_{3}$ with $M_{3} \leq M_{2}$. The number of classes $M_{3}$ as well as the number of regions in $T_{f_{3}}$ is often reduced. In the example in the Fig. 1, the number of classes 90 in $T_{f_{2}}$ becomes 73 in $T_{f_{3}}$ and the number of regions 388 in $T_{f_{2}}$ becomes 314 in $T_{f_{3}}$. This procedure is summarized in the Algorithm 3. 
Alkama S., Chahir Y., Berkani D.: Label maps fusion for the marginal...

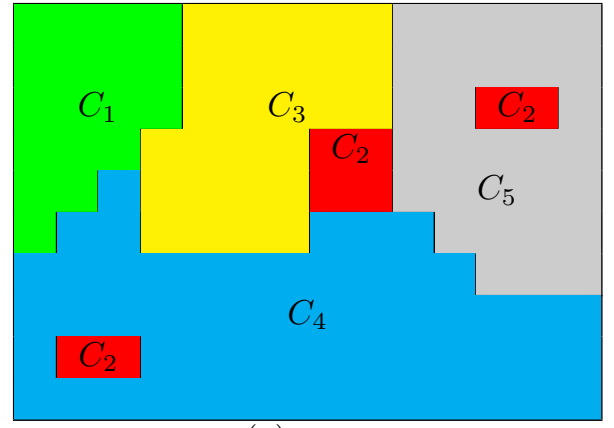

(a)

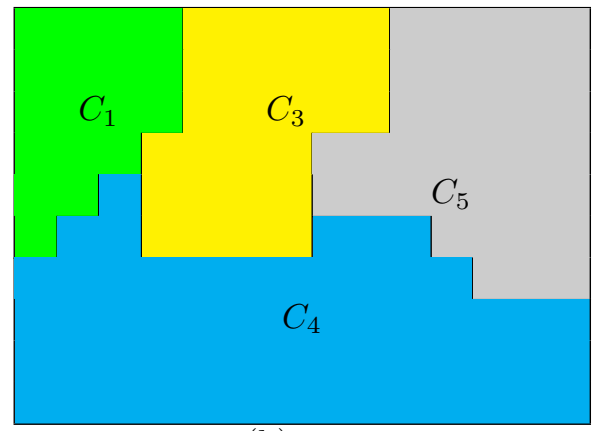

(b)

Fig. 6 Example of elimination of insignificant classes: (a) $T_{f_{2}}$ label map, (b) $T_{f_{3}}$ label map.

The example in the Fig. 6 illustrates the steps of this procedure. The class $C_{2}$, composed of three regions (Fig. 6(a)), is supposed insignificant. Each of these three regions will be treated independently by using the same rule described in the previous section. The rightmost region of $C_{2}$ will obviously assigned to class $C_{5}$, the left-most region to the class $C_{4}$ and the region which is in the center, will be assigned to the class that has a mean color closest to the mean color among the three classes $C_{3}, C_{4}, C_{5}$. In this example it is assumed that it is the class $C_{5}$ (see Fig. 6(b)).

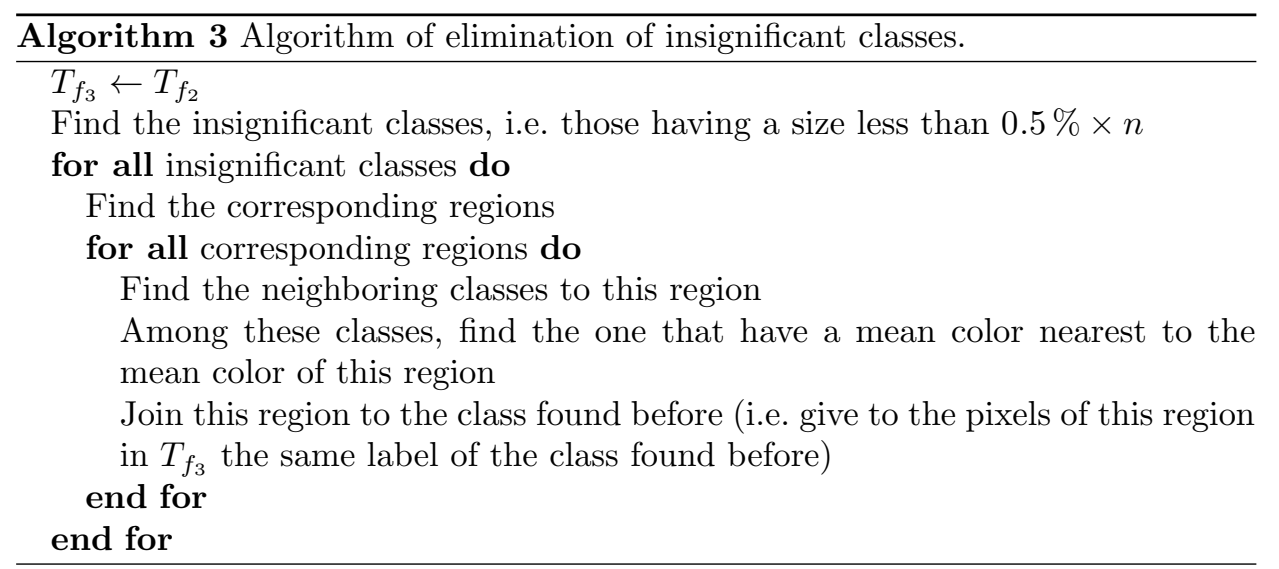

Although only significative classes and significative regions remain at the end of this procedure, the number of classes can be larger than the desired number of classes. To leave to the desired class number, a grouping of classes procedure is performed in the next step. 


\subsection{Grouping of classes}

The last step of the proposed fusion technique aims to group iteratively the $M_{3}$ classes which have the closest mean color until achieve the desired number of classes $K\left(K \leq M_{3}\right)$. This grouping procedure is performed by using the hierarchical agglomerative clustering algorithm [23] which is outlined in Algorithm 4. The results of this procedure is a final segmented image noted $T_{f_{4}}$, see Fig. 1.

The number of classes and regions in $T_{f_{4}}$ is greatly reduced. In the example in the Fig. 1, the number of classes 73 in $T_{f_{3}}$ becomes 7 in the final label map $T_{f_{4}}$ and the number of regions 314 in $T_{f_{3}}$ becomes 86 .

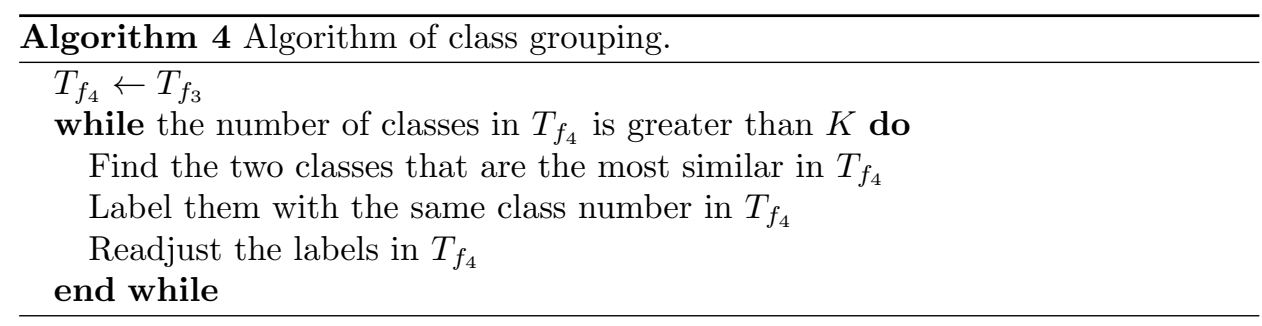

\section{Experiment results and discussion}

In this section, we will evaluate the performance of the proposed marginal multicomponent image segmentation. To assess its effectiveness, we compare the results of this technique with those of the vector segmentation based on the same classification algorithm SOM.

This evaluation is based on the criterion proposed by Borsotti et al. [7]. It is an unsupervised evaluation criterion, which does not require any "ground truth" and the quality of segmentation results is estimated from statistics calculated on each detected area. It is initially developed for the color imagery and can be applied to the multi-spectral imagery by extending the dimension. Moreover the Borsotti et al. function does not require any adjustment parameter, it gives an indication on the quality of the results which is in adequacy with our visual perception and it avoids the inconvenient of its predecessors functions like the Liu and Yang function [34] and their revised version [7,52]. Indeed, the Liu and Yang function tends to evaluate very noisy segmentations favorably when the number of regions built is important and their revised function is not sensitive enough to small segmentation differences $[7,51,52]$.

The Borsotti et al. function is defined by

$$
C_{\mathrm{R}}=\frac{\sqrt{N_{\mathrm{R}}}}{10^{4} n} \sum_{r=1}^{N_{\mathrm{R}}}\left(\frac{E_{r}^{2}}{1+\log \left(\operatorname{card}\left(B_{r}\right)\right)}+\frac{R\left(B_{r}\right)^{2}}{\operatorname{card}\left(B_{r}\right)^{2}}\right),
$$

where $N_{\mathrm{R}}$ is the number of regions obtained in the segmented image, $n$ is the image size, $E_{r}$ the sum of distances between the pixels of the region $B_{r}$ in input image and the multi-component values attributed to the region $B_{r}$ in the segmented image and $R\left(B_{r}\right)$ is the number of regions having exactly an area equal to that of $B_{r}$. 
Alkama S., Chahir Y., Berkani D.: Label maps fusion for the marginal...

The first term in the sum is high only for the non-homogeneous regions (typically, large ones), while the second is high only for the regions whose area $B_{r}$ is equal to the area of many other regions in the segmented image (typically, small ones). This function penalizes the segmentation that produces many regions and having non-homogeneous regions. The lower the $C_{\mathrm{R}}$ value, the better the segmentation.

Two types of multi-component images are used to conduct this evaluation. The first one contains color images which are presented by three color components. The second type is constituted of one multi-spectral image coming from Meteosat MSG2 satellite. The components of this image correspond to five infra-red channels of this satellite (see [36] and [1]).

Fig. 7 shows the results of the segmentation of six color images (one synthetic and five real). The original color images are presented on the first column, the results of the vector segmentation are presented on the second column and those of the proposed marginal segmentation are given on the last column.

Fig. 8 shows the results of the segmentation of a Meteosat MSG2 satellite image. This image describes the north-west African region. It presents different cloudy areas and non-cloudy areas (lads, sea, ...). The five infra-red channels of this image are displayed in gray levels (see Fig. 8(a) to Fig. 8(e)). The segmented image by the vector approach is shown in Fig. 8(f) and the one provided by our method is given in the Fig. $8(\mathrm{~g})$. The segmentation results are displayed with false colors. Let us note that the colors are affected randomly to classes, this implies that the same color in the segmented images obtained by the marginal and vector approaches does not correspond necessarily to the same class.

The values of the Borsotti function are estimated on all test images and are grouped in the Tab. I. The number of classes $K$ as well as the number $N_{\mathrm{R}}$ of the detected regions by each segmentation method are specified in Tab. I.

\begin{tabular}{lccccc}
\hline & Class number & \multicolumn{2}{c}{ Borsotti's $C_{\mathrm{R}}$} & \multicolumn{2}{c}{ Number of regions $N_{\mathrm{R}}$} \\
Image & $K$ & Vector & Marginal & Vector & Marginal \\
\hline Synthetic & 6 & 517 & 44 & 779 & 6 \\
Peppers & 7 & 527 & 317 & 1079 & 86 \\
Plane & 2 & 584 & 216 & 34 & 4 \\
Flowers & 5 & 3628 & 1210 & 1108 & 61 \\
Church & 6 & 1681 & 295 & 1505 & 36 \\
Elephant & 7 & 9948 & 778 & 4330 & 75 \\
Meteosat & 15 & 10311 & 243 & 7687 & 891 \\
\hline
\end{tabular}

Tab. I Values of Borsotti criterion and number of detected regions. Vector: Vector segmentation. Marginal: Proposed marginal segmentation.

From the results of vector and marginal segmentations displayed in Fig. 7 and Fig. 8, we can see that the proposed marginal technique provides good results. Indeed, for example, in the case of the synthetic color image, many pixels of the orange square region are badly assigned to the yellow and pink regions when we use the vector segmentation, whereas the proposed marginal segmentation affects them correctly. We also find that the little green circle is confused with the large 


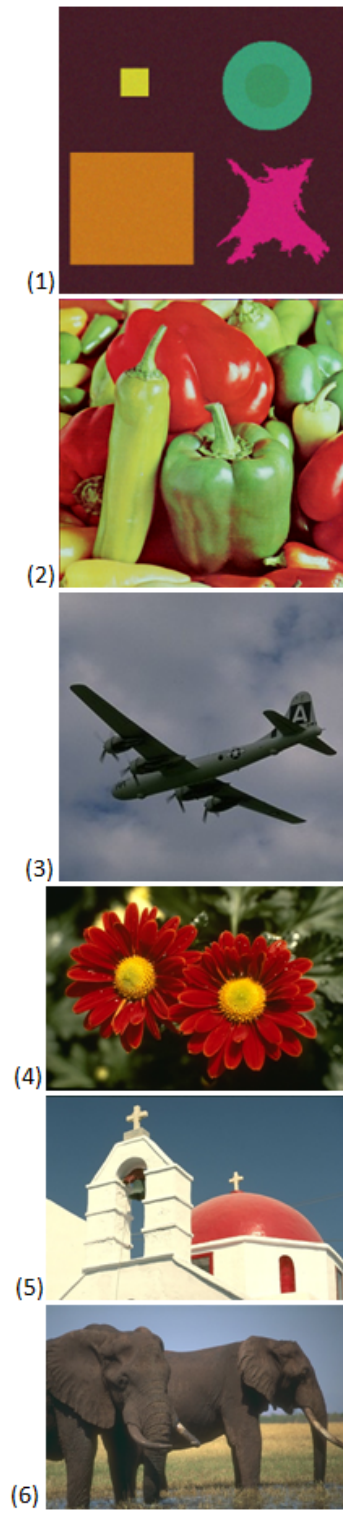

(a)
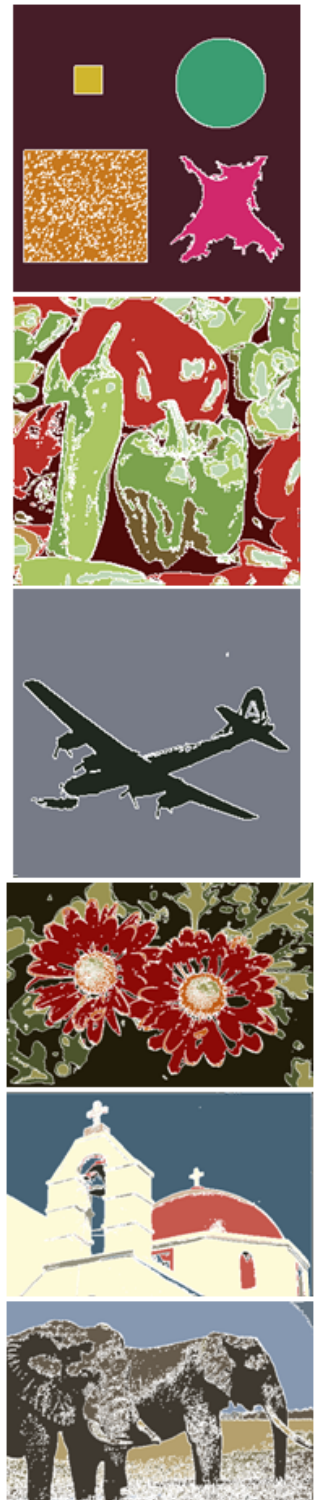

(b)
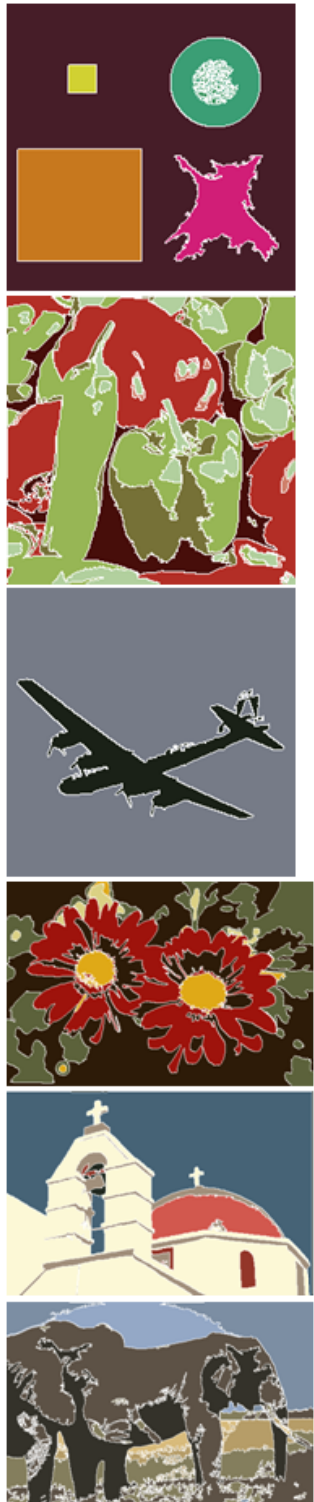

(c)

Fig. 7 The results of the color image segmentation. Rows: (1) Synthetic, (2) Peppers, (3) Plane, (4) Flowers, (5) Church, (6) Elephants. Columns: (a) Original images, (b) Vector segmentation, (c) Proposed marginal segmentation.

circle in the case of the vector segmentation, whereas in the case of the marginal segmentation just some pixels of this object are misclassified. We can also cite the case of the plane image where a part of the sky is badly detected by the vector segmentation and it is convenably detected by the marginal segmentation. The 
Alkama S., Chahir Y., Berkani D.: Label maps fusion for the marginal...

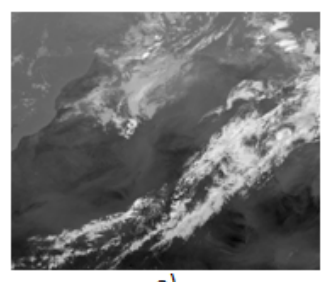

a)

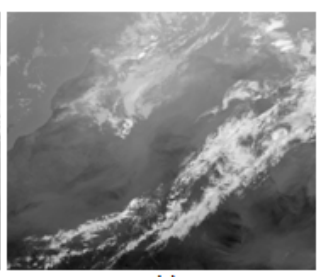

b)

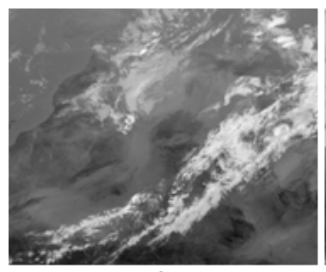

c)

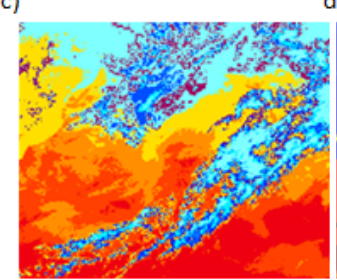

f)

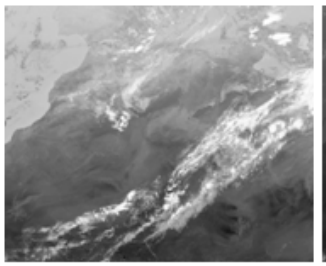

d)

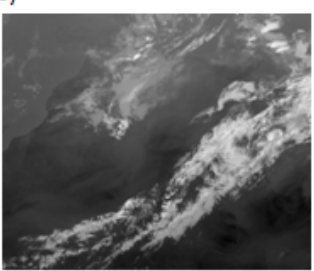

e)

Fig. 8 The results of the segmentation in 15 classes of the Meteosat image taken at noon December 15, 2006. The original image components: (a) IR8.7 (b) IR9.7 (c) IR10.8 (d) IR12.0 e) IR13.4. Segmentation results: (f) Vector segmentation (g) Marginal segmentation.

improvement of results of the marginal segmentation comparatively to the vector segmentation is also observed on the other images in Fig. 7. This observation is confirmed by the values of Borsotti criterion $C_{\mathrm{R}}$ given in Tab. I, where they are always better for the marginal segmentation. As regards the Meteosat satellite image, we can see that the clouds are well detected in both cases (the different blue ones and the purple one in the case of the vector segmentation and the different blue ones without the darkest and the clear green in the case of the marginal segmentation). However, we remarque, in the case of the vector segmentation, that many low size regions (even that have one pixel size) are detected. This is a disadvantage which is eliminated by the proposed marginal segmentation. The Borsotti function given for this image in Tab. I is also clearly improved by our marginal segmentation.

In the marginal approach, the dependence between the components is completely ignored. At prior, we can expect to best results with the vector segmentation since all the dependencies between the components are taken into account. However, after experiment we found, in spite of this, the proposed marginal technique provides better results than those of the vector segmentation. We conclude that the developed fusion technique is effective due to different processing steps notably, to the step where the small regions are eliminated. These regions are 
assimilated to noise and, as stipulated by Borsotti et al. [7], they penalize their function. Effectively, when we observe the results of the Tab. I, we note that the number of detected regions $N_{\mathrm{R}}$ is reduced in the marginal segmentation comparatively to the vector segmentation. The proposed fusion technique always removes the insignificant regions and classes and reveals only the most important regions and classes.

The originality of the proposed fusion procedure lies in the two procedures of elimination of insignificant regions and classes and especially how the pixels belonging to these removed regions and classes are assigned to the remaining significant classes.

Indeed, the elimination of the insignificant regions and classes allows to find, at the end of the fusion procedure and after the class grouping step, only the significant classes. For example, if these two steps are not performed, some small classes (which correspond to noise) can be founded in the final result and several classes corresponding to various areas of interest can be grouped, giving then a bad segmentation.

A priori, we would think that the elimination of the insignificant classes may be sufficient, but this can leave small regions (which correspond to the noise). Indeed, a significant class, which will not be eliminated by this step, can be formed by several regions, where some of them may be insignificants.

The succession in which the insignificant regions and classes are eliminated does not matter because the final result is substantially identical. Indeed, in these two steps, the elimination is performed by assigning the regions to the appropriate classes.

During the elimination of insignificant classes, each region of these classes is treated independently. The pixels of these regions are not necessarily assigned to the same significant class. Indeed, if we observe the example in Fig. 6, it seems obvious that the three regions of the insignificant class $C_{2}$ will be added to different significant classes.

In this paper, we compared the results obtained by using the proposed marginal fusion procedure with those of the vector segmentation to establish the effectiveness of the proposed fusion method for the marginal segmentation. It is obvious that segmentation algorithm used in the marginal segmentation and in the vector segmentation must be the same. In this paper, the segmentation algorithm is performed by using Self Organizing Map (SOM) and fine-tuned with Hierarchical Agglomerative Clustering (HCA) algorithm. Others classical classification algorithms like Expectation-Maximization (EM), Fuzzy C-Means (FCM), $k$-means, Markov Random Field (MRF) can also be used. Preliminary works, which are not given in this paper, show the efficiency of the SOM comparatively to the above cited algorithms. Other methods using the SOM algorithm combined with another algorithm such as Fuzzy C-Means (FCM) [40], Learning Vector Quantization (LVQ) [13], Hybrid Genetic Algorithm (HGA) [4] can also be used. However, it is evident that when one method of segmentation provides better results in vector segmentation, it provides also better results in marginal segmentation but the comparison of the segmentation methods is not the purpose of this paper. 
Alkama S., Chahir Y., Berkani D.: Label maps fusion for the marginal...

\section{Conclusion}

In this paper, we used two approaches to segment multi-components images, the marginal approach and the vector approach. The same SOM algorithm is used to segment the components of the image in the case of the marginal approach and the multi-component image in the case of the vector approach. In the marginal approach, we developed a new method for the fusion of label maps.

To assess the effectiveness of the proposed marginal segmentation, several tests are performed on two types of the multi-components images, color and satellite images.

The quantitative evaluation, performed on these images, shows that the marginal technique proposed provides better results comparatively to the vector approach.

Although, in its structure, the marginal approach ignores the dependency that exist between the different components, neglecting therefore the information that can participates in improving the performance of treatments, we observe that the results obtained by this proposed marginal approach are much better than those obtained by the vector approach. We conclude that the developed fusion technique is effective due to its different processing steps, notably to the manner in which the small and insignificant regions and classes, produced by the superposition step, are eliminated then reassigned.

\section{References}

[1] ALKAMA S., CHAHIR Y., BERKANI D. Markovian approach using several Gibbs energy for remote sensing images segmentation. Analog Integrated Circuits and Signal Processing. 2011, 69(1), pp. 39-47, doi: 10.1007/s10470-011-9631-8.

[2] ANITHA J., JUDE HEMANTH D. An efficient Kohonen-fuzzy neural network based abnormal retinal image classification system. Neural Network World. 2013, 23(2), pp. 149-167, doi: 10.14311/NNW.2013.23.011.

[3] ARAUJO A.R.F., COSTA D.C. Local adaptive receptive field self-organizing map for image color segmentation. Image and Vision Computing. 2009, 27(9), pp. 1229-1239, doi: 10.1016/j.imavis.2008.11.014.

[4] AWAD M., CHEHDI K., NASRI A. Multicomponent image segmentation using a genetic algorithm and artificial neural network. IEEE Geosience and Remote Sensing Letters. 2007, 4(4), pp. 571-575, doi: 10.1109/LGRS.2007.903064.

[5] AWAD M. Segmentation of satellite images using Self-Organizing Maps. In: G.K. MATSOPOULOS, ed. Self-Organizing Maps. InTech, 2010, doi: 10.5772/3473.

[6] BHATTACHARYA P., BISWAS A., MAITY S.P. Wavelets-based clustering techniques for efficient color image segmentation. In: M.K. Kundu, D.P. Mohapatra, A. Konar, A. Chakraborty. Advanced Computing Networking and Informatics - Volume 1. Smart Innovation Systems and Technologies (series title). 2014, 27, pp. 237-244, doi: 10.1007/978-3-319-07353-8_28.

[7] BORSOTTI M., CAMPADELLI P., SCHETTINI R. Quantitative evaluation of color image segmentation results. Pattern Recognition Letters. 1998, 19(8), pp. 741-747, doi: 10.1016/S0167-8655(98)00052-X.

[8] BUSIN L., VANDENBROUCKE N., MACAIRE L. Color spaces and image segmentation. Advances in Imaging and Electron Physics. USA: Elsevier Inc., 2008, 151, pp. 65-168, doi: 10.1016/S1076-5670(07)00402-8.

[9] CAPELlE A.-S., COLOT O., Fernandez-Maloigne C. Evidential segmentation scheme of multi-echo MR images for the detection of brain tumors using neighborhood information. Information Fusion. 2004, 5(3), pp. 203-216, doi: 10.1016/j.inffus.2003.10.001. 
[10] CHARRIER C., LEZORAY O. Segmentation d'images couleur par la théorie de l'évidence. In: Proceedings of COmpression et REprésentation des Signaux Audiovisuels (CORESA 2005), Rennes, France. 2005, pp. 291-296.

[11] CHEN K., MA Y., LIU J. Segmentation by fusion of self-adaptive SFCM cluster in multicolor space components. International Journal of Image Processing. 2012, 6(2), pp. 157-166.

[12] CHENG H.D., JIANG X.H., SUN Y., WANG J. Color image segmentation: advances and prospects. Pattern Recognition. 2001, 34(12), pp. 2259-2281, doi: 10.1016/S0031-3203(00)0149-7.

[13] DEMIRHAM A., TOUR M., GULER I. Segmentation of tumor and edema along with healthy tissues of brain using wavelets and neural networks. IEEE Journal of Biomedical and health Informatics. 2014, 19(4), pp. 1451-1458, doi: 10.1109/JBHI.2014.2360515.

[14] DEMPSTER A.P. A generalization of bayesian inference. Journal of the Royal Statistical Society. Series B (Methodological). 1968, 30(2), pp. 205-247.

[15] FRANEK L., DURATE ABDALA D., VEGA-PONS S., JIANG X. Image segmentation fusion using general ensemble clustering methods. Lecture Notes in Computer Science. 2011, 6495, pp. 373-384, doi: 10.1007/978-3-642-19282-1_30.

[16] GIANNAROU S., STATHAKI T. Fusion of edge maps using statistical approaches. In: T. Stathaki, ed. Image fusion, algorithms and application. Academic Press, 2008, pp. 273-298, doi: 10.1016/B978-0-12-372529-5.00006-8.

[17] HARRABI R., BRAIEK E.B. Color image segmentation using multi-level thresholding approach and data fusion techniques: application in the breast cancer cells images. EURASIP Journal on Image and Video Processing. 2012, 2012(11), doi: 10.1186/1687-5281-2012-11.

[18] HARRABI R., BEN BRAIEK E. Color image segmentation by multilevel thresholding using a two stage: Optimization approach and fusion. International Journal of Engineering and Innovative Technology. 2014, 3(11), pp. 14-20.

[19] HEMA RAJINI N., BHAVANI R. Automatic MR Brain tumor detection using possibilistic CMeans and K-means clustering with color segmentation. International Journal of Computer Applications. 2012, 56(2), pp. 11-17, doi: 10.5120/8862-2825.

[20] HEMELATHA M., JAGADEESHKANNAN R. Segmentation by blended partitional clustering for different color spaces. International Journal of Recent Trends in Engineering. 2009, 1(1), pp. 630-634, doi: 01.IJRTET.2009.01.01.261.

[21] HOLOTA R. Colour image recognition based on single-layer networks of Min/Max nodes. Neural Network World. 2012, 22(4), pp. 395-405, doi: 10.14311/NNW.2012.22.024.

[22] IOANNOU S., AVRITHIS Y., STAMOU G., KOLLIAS S. Fuzzy data fusion for multiple cue image and video segmentation. Studies in Fuzziness and Soft Computing. 2003, 122, pp. 195-215, doi: 10.1007/978-3-540-36420-7_9.

[23] JAIN A.K., DUBES R.C. Algorithms for clustering data. Prentice Hall, 1988.

[24] JAWALE S.H., BAVASKAR A.B. Fusion based edge detection and segmentation of color spaces. International Journal of Advanced Information and Communication Technology. 2014, 1(7), pp. 601-604, doi: 01.0401/ijaict.2014.07.16.

[25] JIANG Y., ZHOU Z.-H. SOM ensemble-based image segmentation. Neural Processing Letters. 2004, 20(3), pp. 171-178, doi: 10.1007/s11063-004-2022-8.

[26] JUANG L.-H., WU M.-N. MRI brain lesion image detection based on colorconverted K-means clustering segmentation. Measurement. 2010, 43(7), pp. 941scie, doi: 10.1016/j.measurement.2010.03.013.

[27] KANIMOZHI M., HIMA BINDU CH. Brain MR image segmentation using self organizing map. International journal of advanced research in Computer and Communication Engineering. 2013, 2(10), pp. 3968-3973.

[28] KOHONEN T. Self organization and associative memory. Berlin, Heidelberg: Springer, 1984.

[29] KOHONEN T. Self Organizing Maps. 3rd. ed. Springer, 2000.

[30] KUDELKA M., HORAK Z., VOZENILEK V., SNASEL V. Orthophoto feature extraction and clustering. Neural Network World. 2012, 22(2), pp. 103-121, doi: 10.14311/NNW.2012.22.007. 
Alkama S., Chahir Y., Berkani D.: Label maps fusion for the marginal...

[31] KURUGOllu F., SANKUR B., HARMANCI A.E. Color image segmentation using histogram multithresholding and fusion. Image and Vision Computing. 2001, 19(13), pp. 915928, doi: 10.1016/S0262-8856(01)00052-X.

[32] LEZORAY O., CHARRIER C. Segmentation d'images couleur par coalescence non supervisée d'histogrammes 2D et fusion de régions selon la théorie de Dempster-Shafer. Traitement du Signal. 2004, 21, pp. 605-621.

[33] LEZORAY O., CHARRIER C. Color image segmentation using morphological clustering and fusion with automatic scale selection. Pattern Recognition Letters. 2009, 30(4), pp. 397-406, doi: $10.1016 / \mathrm{j}$.patrec.2008.11.005.

[34] LIU J., YANG Y.-H. Multiresolution color image segmentation. IEEE transaction on Pattern Analysis and Machine Intelligence. 1994, 16(7), pp. 689-700, doi: 10.1109/34.297949.

[35] MAUTNER P., MOUCEK R. Processing and categorization of Czech written documents using neural networks. Neural Network World. 2012, 22(1), pp. 53-66, doi: 10.14311/NNW.2012.22.004.

[36] EUMETSAT. Available from: http://www. eumetsat.int

[37] MiGNOTTE M. Segmentation by fusion of histogram-based K-means clusters in different color spaces. IEEE Transaction in Image Processing. 2008, 17(5), pp. 780-787, doi: 10.1109/TIP.2008.920761.

[38] MignOTTE M., HELOU C. A precision-recall criterion based consensus model for fusing multiple segmentations. International Journal of Signal Processing, Image Processing and Pattern Recognition. 2014, 7(3), pp. 61-82, doi: 10.14257/ijsip.2014.7.3.07.

[39] MITCHELL H.B. Image fusion: theories, thechniques and application. Springer, 2010.

[40] ORTIZ A., PALACIO A.A., GORRIS J.M., RAMIREZ J., SALAS-GONZALEZ D. Segmentation of brain MRI using SOM-FCM-based method and 3D statistical descriptors. Computational and Mathematical Methods in Medicine. 2013, 2013, pp. 12, doi: 10.1155/2013/638563.

[41] PLATANiOTIS K.N., VENETSANOPOULOS A.N. Color image processing and applications. Springer, 2000.

[42] RAMirez C., ARGAEZ M., GUILlEN P., GONZALEZ G. Self organizing maps in seismic image segmentation. Computer Technology and Application. 2012, pp. 624-629. Available from: http://www.davidpublishing.com/davidpublishing/upfile/11/7/2012/ 2012110703267796.pdf

[43] ROSEnFELD A., KAK A.C. Digital Picture Processing. Computer Science and Applied Mathematics. 2nd ed. Academic Press, 1982.

[44] SAMUEL VARAPRASAD RAJU, PRASAD G.S., SRIKANTH Y. Edge detection and segmentation by fusion of histogram-based K-means clusters in different color spaces. Computer Science and Telecommunications. 2013, 38(2), pp. 10-28.

[45] SHAFER G. A Mathematical theory of evidence. Princeton University Press, 1976.

[46] SURESH KUMAR P.S., RAMANA REDDY P. Segmentation by K-means clustering indifferent color spaces. International Journal of Advances in Electronics Engineering. 2011, 1(1), pp. 401-407.

[47] WANG X., DU J., WU S., LI X., LI F. Cluster ensemble-based image segmentation. International Journal of Advanced Robotic Systems. 2013, 10, pp. 11, doi: 10.5772/56769.

[48] WEHRENS R. Self-organising maps for image segmentation. Advances in Data Analysis, Data Handling and Business Intelligence. Studies in Classification, Data Analysis, and Knowledge Organization (series title). 2010, pp. 373-383, doi: 10.1007/978-3-642-01044$6 \_34$.

[49] XUE Z., JIA C. A new method of color map segmentation based on the self-organizing neural network, emerging intelligent computing technology and applications with aspects of artificial intelligence. Emerging Intelligent Computing Technology and Applications. With Aspects of Artificial Intelligence. Lecture Notes in Computer Science (series title). 2009, 5755, pp. 417-423, doi: 10.1007/978-3-642-04020-7_44. 
[50] YU Z., OSCAR C., ZOU R., YU W., TIAN J. An adaptive unsupervised approach toward pixel clustering and color image segmentation. Pattern Recognition. 2010, 43(5), pp. 18891906, doi: $10.1016 /$ j.patcog.2009.11.015.

[51] ZHANG H., FRITTS J.E., GOLDMAN S.A. Image segmentation evaluation: A survey of unsupervised methods. Computer Vision and Image Understanding. 2008, 110(2), pp. 260280, doi: 10.1016/j.cviu.2007.08.003.

[52] ZHANG Y.J. A survey of evaluation methods of image segmentation. Pattern Recognition. 1996, 29(8), pp. 1335-1346, doi: 10.1016/0031-3203(95)00169-7. 